\title{
MIDDLE CRANIAL FOSSA ARACHNOID CYSTS COMPLICATED WITH SUBDURAL COLLECTIONS
}

\author{
Felipe Slaviero', Leonardo Frighetto ${ }^{2}$, Nério Dutra Azambuja Júnior², \\ Lucas Schirmer Martins', Rafael D’Agostini Annes ${ }^{2}$, José Ricardo Vanzin ${ }^{2}$
}

Intracranial arachnoid cysts are congenital collections of fluid that develop within the arachnoid membrane because of splitting or duplication of this structure. It corresponds to $1 \%$ of all non-traumatic intracranial mass lesions ${ }^{1}$. Arachnoid cysts most frequently occur in the middle cranial fossa, followed by the posterior fossa, convexity, and suprasellar region. Middle cranial fossa cysts are more often associated with subdural hematoma, subdural hygroma, and intracystic hemorrhage. Usually, the patients present with signs and symptoms of intracranial hypertension, mainly headache and vomiting ${ }^{2}$.

We report two cases of arachnoid cysts associated with concurrent subdural collections, describing their clinical presentation, radiographic findings and neurosurgical management.

\section{CASES}

Case 1

A 15-year-old male patient presented with a 3-day history of headache and vomiting. He reported an episode of mild head trauma without loss of consciousness four days before the ad- mission. There was no history of drugs, alcohol abuse or previous neurological illness. The Glasgow coma score was 14 (confused conversation). Pupil reactions were normal and there was no other abnormality in the neurologic examination. Computed tomography (CT) revealed an arachnoid cyst in the left middle cranial fossa (Fig 1A) and a low-density crescentic collection across the entire left hemispheric convexity, corresponding to a chronic subdural hematoma, with signs of acute hemorrhage (Fig 1B). The patient underwent surgical treatment with evacuation of the subdural hematoma and endoscopic fenestration of the cyst to obtain communication with the chiasmatic cistern. The recovery was uneventful and an eight months follow-up CT showed a residual non-hypertensive cyst. There was no evidence of any subdural collection.

\section{Case 2}

A healthy 5-year-old male patient presented with a six-day history of intense headache and drowsiness. There was no history of head trauma, hematologic disease or use of any medication that could have possibly caused hemorrhage. The physical examination demonstrated a localized left temporal skull bulging. The
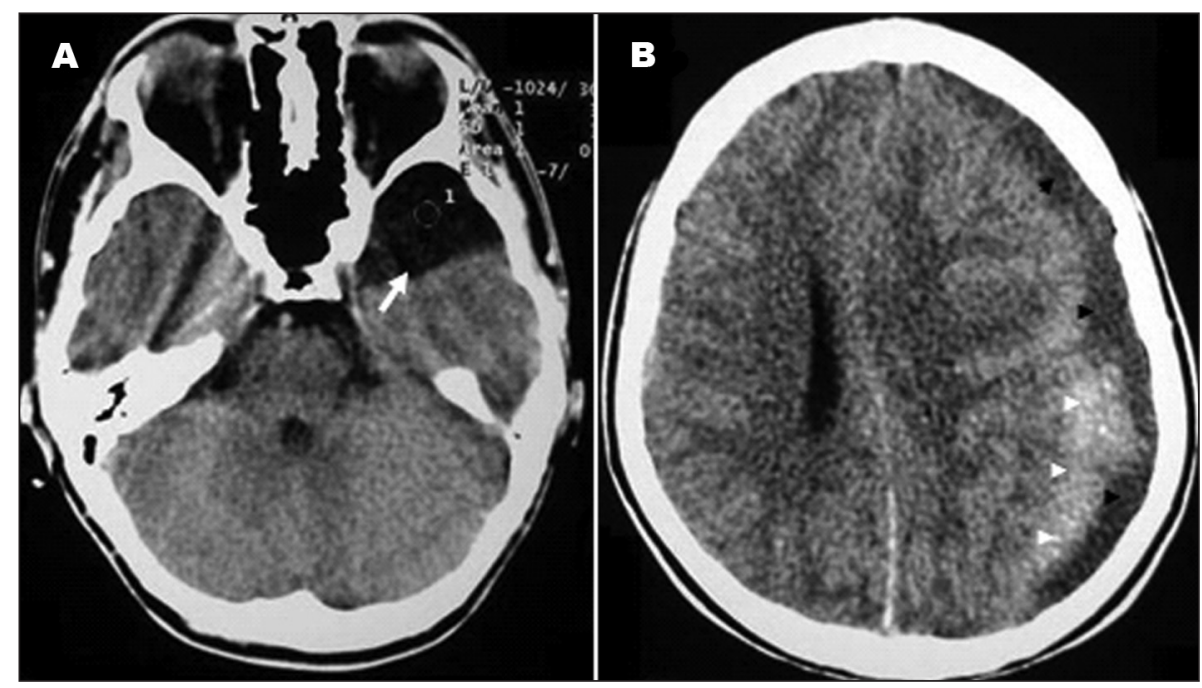

Fig 1. (A) Axial CT showing an arachnoid cyst in the left middle cranial fossa (arrow). (B) Chronic subdural hematoma (black arrowheads), with signs of acute hemorrhage (white arrowheads).

\section{CISTOS DA ARACNÓIDE DA FOSSA CRANIANA MÉDIA COMPLICADOS COM COLEÇÕES SUBDURAIS}

Neurology and Neurosurgery Service, Hospital São Vicente de Paulo, Passo Fundo RS, Brazil: 'Medical Student, Passo Fundo University School of Medicine; ${ }^{2}$ Neurosurgeon.

Received 1 July 2008. Accepted 15 September 2008.

Dr. Felipe Slaviero - Edifício das Clínicas - Rua Teixeira Soares, 885 / 10ªndar - 99010-901 Passo Fundo RS - Brasil. E-mail: felipeslavier@yahoo.com.br. 

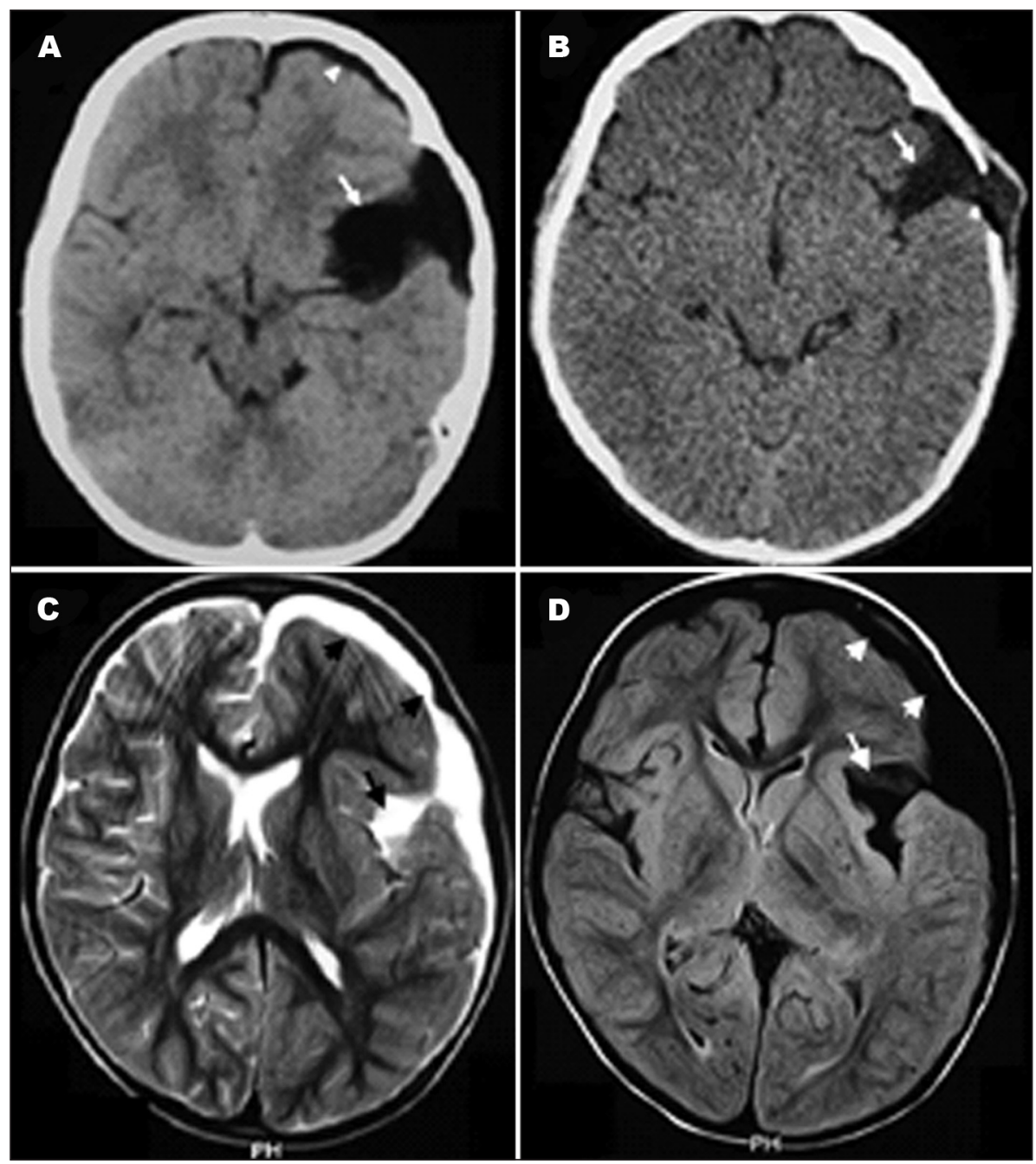

Fig 2. (A) Preoperative CT demonstrating an arachnoid cyst (arrow) with subdural hygroma (arrowhead). (B) Postoperative $C T$ demonstrating the temporal craniotomy and a small residual cyst (arrow). (C, D) T2-weighted and FLAIR MRI showing an arachnoid cyst (arrow) with subdural hygroma (arrowheads) causing mass effect and contralateral shift of the midline.

neurological examination was normal. CT disclosed a subdural hygroma in the left fronto-temporal convexity and a left middle fossa arachnoid cyst (Fig 2A). Magnetic resonance imaging (MRI) demonstrated the presence of a cystic lesion in the left middle cranial fossa with high signal intensity on T2-weighted image (Fig $2 \mathrm{C}$ ), and low signal intensity on fluid attenuation inversion recovery image (FLAIR) (Fig 2D). The patient was submitted to surgical evacuation of the subdural hygroma and endoscopic fenestration of the cyst. Complete postoperative recovery was achieved and the patient has remained asymptomatic during an eight months follow up period. A CT performed eight months after treatment showed no recurrence of extra-axial collections and a decrease in cyst size (Fig 2B).

\section{DISCUSSION}

Arachnoid cysts are developmental anomalies often diagnosed in the childhood and remain asymptomatic until they compress the adjacent neural structures, obstruct the cerebrospinal fluid (CSF) circulation or develop a hemorrhagic complication ${ }^{3}$. There is a predilection for the left side and the male sex. The natural history of arachnoid cysts is not well defined, but large cranial middle fossa cysts are more likely to be symptomatic and appear to have the highest risk of undergoing significant expansion ${ }^{4}$.

Symptomatic arachnoid cysts may manifest as generalized cranial enlargement, localized cranial bulging, increased intracranial pressure, seizures, psychomotor retardation, focal neurological deficits, hydrocephalus, and dementia ${ }^{5}$. The occurrence of secondary lesions may include subdural hematoma, subdural hygroma and intracystic hemorrhage, all of which may occur following head trauma or spontaneously.

The annual risk of hemorrhage within or around these cysts is reported to be less than $0.1 \%^{6}$, although there is an expected cumulative risk of up to $20 \%$ of subdural hematoma, mainly complicating those cysts in the middle cranial fossa ${ }^{5}$. A CT or a MRI can provide the diagnosis of an arachnoid cyst and its complications. On CT, arachnoid cysts appear as a well-defined lesion, with the same density of the CSF and without contrast enhancement. MRI is 
the neuroimaging of choice and shows more properly the arachnoid cyst and the simultaneous complications.

The best neurosurgical approach for the treatment of intracranial arachnoid cysts remains controversial. The options include an expectant management, cyst shunting, craniotomy for cyst fenestration, deviation of cyst fluid to another intracranial space and endoscopic fenestration? ${ }^{7}$.

Levy et al. ${ }^{7}$ in a series of 50 patients with arachnoid cysts in the middle cranial fossa who underwent cyst fenestration via a microsurgical craniotomy approach showed that $82 \%$ of patients demonstrated decrease in cyst size and $95 \%$ of the patients had exhibited clinical improvement in at least one of their preoperative symptoms. A study of 22 patients with intracranial arachnoid cysts treated with neuroendoscopic fenestration between the cyst and the cerebral cistern demonstrated clinical improvement in all patients. This result suggests that neuroendoscopic cystic fenestration is effective and associated with low mortality and morbidity rates ${ }^{8}$.

Greenfield et al., reported a series of 33 patients who underwent endoscopic cyst fenestration for the treatment of intracranial arachnoid cysts, symptomatic improvement was achieved in $97 \%$ of the patients, with no surgery-related morbidities or deaths ${ }^{9}$. Other several series demonstrated that endoscopic fenestration is an effective and secure treatment for symptomatic arachnoid cysts $^{10}$. In the present study, both patients submitted to surgical treatment with endoscopic fenestration of the cyst evolved with resolution of the subdural collection, improvement of symptoms and reduction of the cyst size in the follow-up CT.

According to the literature and with the results of the present report, endoscopic procedures are a useful alternative to the traditional microsurgical approach or shunting, for the treatment of arachnoid cysts. The lack of reported cases about management of arachnoid cysts complicated with subdural collections makes the best surgical treatment still controversial. We report these two cases to suggest that surgical evacuation of the hematoma followed by an endoscopic cyst fenestration is a minimally invasive, safe and effective approach for the treatment of middle cranial fossa arachnoid cysts complicated with subdural collections.

ACKNOWLEDGEMENT - We thank Senair Alberto Ambros, MD, for his expert assistance in neuroradiology.

\section{REFERENCES}

1. Iaconetta G, Esposito M, Maiuri F, Cappabianca P. Arachnoid cyst with intracystic haemorrhage and subdural haematoma: case report and literature review. Neurol Sci 2006;26:451-455.

2. Mori K, Yamamoto T, Horinaka N, Maeda M. Arachnoid cyst is a risk factor for chronic subdural hematoma in juveniles: twelve cases of chronic subdural hematoma associated with arachnoid cyst. J Neurotrauma 2002;19:1017-1027.

3. Donaldson JW, Brown ME, Luerssen TG. Arachnoid cyst rupture with concurrent subdural hygroma. Pediatr Neurosurg 2000;32:137-139.

4. Becker T, Wagner M, Hofmann E, Warmuth-Metz M, Nadjmi M. Do arachnoid cysts grow? A retrospective CT volumetric study. Neuroradiology 1991;33:341-345.

5. Prabhu VC, Bailes JE. Chronic subdural hematoma complicating arachnoid cyst secondary to soccer-related head injury: case report. Neurosurgery 2002;50:195-198.

6. Parsch CS, Krauss J, Hofmann E, Meixensberger J, Roosen K. Arachnoid cysts associated with subdural hematomas and hygromas: analysis of 16 cases, long-term follow-up, and review of the literature. Neurosurgery 1997;40:483-490.

7. Levy ML, Wang M, Aryan HE, Yoo K, Meltzer H. Microsurgical keyhole approach for middle fossa arachnoid cyst fenestration. Neurosurgery 2003;53:1138-1145.

8. Huang Q, Wang D, Guo Y, Zhou X, Wang X, Li X. The diagnosis and neuroendoscopic treatment of noncommunicating intracranial arachnoid cysts. Surg Neurol 2007;68:149-154.

9. Greenfield JP, Souweidane MM. Endoscopic management of intracranial cysts. Neurosurg Focus 2005;15;19:E7.

10. Elhammady MS, Bhatia S, Ragheb J. Endoscopic fenestration of middle fossa arachnoid cysts: a technical description and case series. Pediatr Neurosurg 2007;43:209-215. 